\title{
Airplane Capabilities: Translating Non-Normal Information for Operational Decision-Making
}

\author{
Randall J. Mumaw ${ }^{1}$ \\ NASA Ames Research Center, Mail Stop 262-4, P.O. Box 1, Moffett Field, CA 94035-0001, USA \\ Michael Feary ${ }^{2}$ \\ NASA Ames Research Center, Mail Stop 262-4, P.O. Box 1, Moffett Field, CA 94035-0001, USA \\ and \\ Lars Fucke ${ }^{3}$ \\ Diehl Aviation, Am Flugplatz, 88471 Laupheim, Germany
}

\begin{abstract}
We consider how a jet transport airplane interface supports the flight crew in managing airplane system failures (or non-normals) for continued safe flight and landing. The existing state of the art starts with a list of airplane system component failures and asks the flight crew to determine, with the help of non-normal procedures, the operational consequences of those failures. As airplane systems become more complex and interconnected, the flight crew's ability to determine operational consequences will become inadequate. We describe an approach that attempts to translate airplane system failures directly into airplane "capabilities," which is a set of basic airplane functions, such as the ability to stop after landing. This paper describes the overall framework for supporting flight crews in operational decision making and the initial efforts to develop a language and display concepts.
\end{abstract}

\section{Nomenclature}

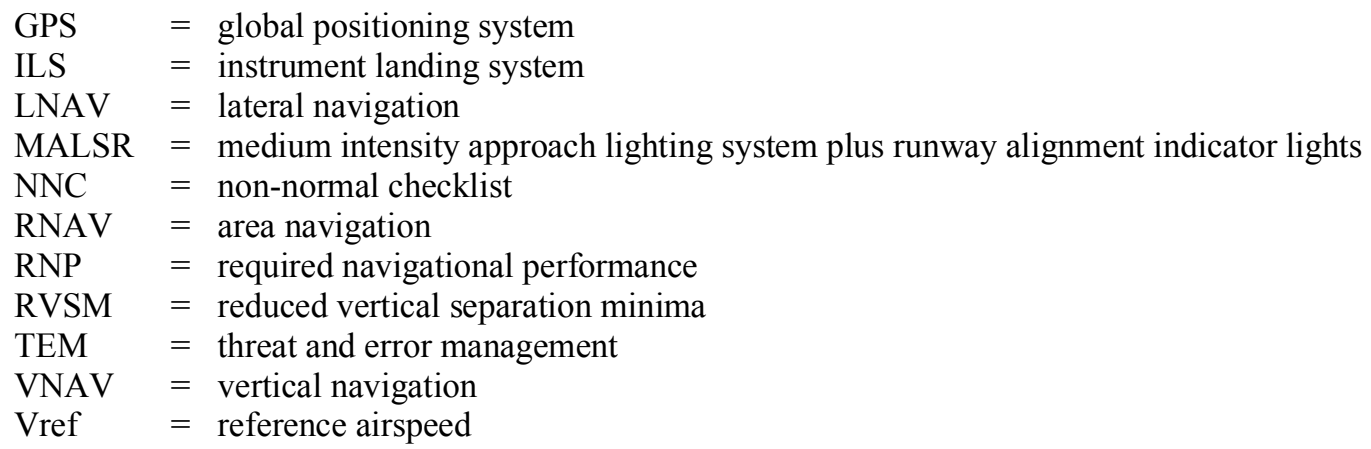

\section{Introduction}

Throughout the history of commercial aviation, the approach to managing airplane system failures has been tied to sensing and reporting on failures of physical airplane components (e.g., a hydraulic system or an electrical bus). This approach requires the flight crew, with the aid of procedures, to sort out how the airplane is affected in terms of continued safe flight and landing. This can be a complex task for flight crews, and, in a number of cases, flight crews have been unable to anticipate fully the consequences (e.g., AAL 268; https://www.ntsb.gov/ layouts/ ntsb.aviation/brief2.aspx?ev id=20081007X03940\&ntsbno=CHI08IA292\&akey=1).

\footnotetext{
${ }^{1}$ Senior Research Associate, San Jose State University Research Foundation

${ }^{2}$ Aerospace Technologist, Human-Systems Integration

${ }^{3}$ Sr. Systems Specialist, R\&T and Innovation
} 
The flight crew's ability to transition effectively from failures in airplane system components to operational decisions is being adversely affected by the increasing complexity and interconnectedness of systems in recently developed airplanes. Although more intensive pilot training could reduce the impact of these changes, it is highly unlikely that airplane systems training will be increased sufficiently to support pilots in reasoning through airplane system failures.

A more-promising approach is to remove the need for pilots to reason from component failures to operational consequences. We developed a prototype that translates physical system components directly into airplane "capabilities," which is a set of basic airplane functions. Ideally, these capabilities, when also combined with other operational information that can be acquired from the world (e.g., current weather, changes to infrastructure), can present information to the flight crew that is more closely aligned with operational decisions.

\section{Flight Crew Response to Non-Normals}

When airplane system failures occur, the flight crew response needs to address the following:

1. Manage any immediate threats to the flight. The elements of this activity are to identify the immediate threats (such as a stall or depressurization), take action to remove them or manage them, and achieve a safe, stable, and flyable airplane.

2. Reconfigure airplane systems. This activity can include containing failures, restoring system functions, and, mitigating system failures. Note that there may be limited ability to achieve these objectives.

3. Revise the mission, as needed. The final consideration in responding to airplane system failures are changes to the operational limitations of the airplane. The airplane system failures may lead to, for example, limitations in airspeed, flap settings, or the need to manually deploy the landing gear. When failures are more significant, there may be a need to revise the mission; that is, it may not be possible to fly safely to the planned destination. The flight crew needs to determine if there is a need to revise the mission, and if so, in what way. Further, this assessment can be on-going as there may be changes to weather or, perhaps, continued degradation of airplane systems, such as a fuel leak.

Although all three of these activities are important for responding to non-normals, our work focused on supporting \#2 and \#3; specifically, understanding the implications of the airplane's state and making effective operational decisions. We developed prototype displays in a reconfigurable, jet-transport simulator to better support short- and long-term decision-making.

An input to our work was the current state of the art. We reviewed the most recent Airbus, Boeing, Embraer, Bombardier, and Gulfstream flight decks to understand how their displays and procedures support the transition from initial alerts to making operational decisions. Although these airplanes offer improvements over the airplanes of 30 years ago, they still have these characteristics:

- The vast majority of alerts are presented as system component failures, which are presented singly even when multiple faults occur. That is, there are only a few checklists for combinations of failures. Each detected failure provides a link to a non-normal checklist, and the flight crew performs each checklist in sequence.

- Supporting documentation, such as paper-based or electronic checklists, provides information on changes to operational limitations per component failure; this information is not integrated in order to remove inconsistencies or redundancies across checklists.

- More importantly, the alerting system and associated procedures do not integrate information in direct support of broader mission changes (e.g., diversions; except for more urgent guidance, such as Land as soon as possible).

- There are no formal tools to support integrating other mission-related information, such as weather or changes to infrastructure.

\section{Shift to Airplane Capabilities}

Our objective was to bypass descriptions of system component failures and to present information tied to operational consequences - for example, instead of indicating that there is a failure of speedbrakes, to express the loss in terms of landing distance. Information about specific component failures can have value, especially if there is a need for system reconfigurations to recover functionality or piloting tasks have to be carried out in a different way. However, for this project, we were focused on supporting operational decision making. 
Our development of prototype displays produced several representations (displays) of operational information, as follows:

\section{A. Mission Compatibility}

We argue that the highest-level purpose for the system (airplane) is to complete the planned mission. Thus, the focus of the mission compatibility display is to show how compatible the airplane's current capabilities are with the planned mission, and further, to show if it can fly a specific approach to a specific runway. If the airplane is not compatible with some of the specifics, the display will offer alternative means to complete the mission. The display also integrates the most recent changes to the environment and the infrastructure so that, for example, if an ILS signal is lost on the planned runway, there is a revised assessment of compatibility with the remaining approaches. Compatibility is judged on items such as range, landing distance, and capability for performing specific approaches. Results are displayed graphically to make clear where incompatibilities, if any, lie.

\section{B. Airplane Capabilities}

Airplane capabilities are intended to capture changes to the airplane's ability to perform essential functions, which describe what the airplane can do and where it can go. These capabilities are independent from the mission, i.e., any loss of capability will have to be taken into account by the crew for the remainder of the flight, even if the mission is re-planned. For example, if an airplane cannot maintain a survivable pressure and temperature in the cabin at high altitudes, this will be true no matter the planned destination. Our initial set of airplane capabilities is as follows:

- Autoflight; concerned with changes to the autopilot/autothrottle/autoland capabilities. For example, a change in the ability to perform autoland.

- Navigation; concerned with the navigation system's ability to determine a precise position for the airplane or the direction to go. For example, a change in the ability to maintain RNP.

- Communication; concerned with changes in the ability to communicate with groups. For example, a change in the ability to communicate with the cabin.

- Land; concerned with the ability to land the airplane in the ideal configuration. For example, a weight above max. landing weight.

- Take-off; concerned with the ability to take-off. For example, a failure to configure flaps.

- Approach Access; concerned with the ability to perform arrivals and approaches that may be required. For example, a change in the ability to perform an RNAV approach.

- Airspace Access; concerned with the ability to use certain types of airspace. For example, a change in the ability to use RVSM airspace.

- Cabin/Cargo Environment; concerned with the ability to manage temperature, pressure, and air flow in the cabin or cargo area. For example, a change in the ability to control cabin pressure.

- Ice Protection; concerned with changes in the ability to protect against icing. For example, a change in the ability to keep ice off the wing.

- Landing Distance; concerned with capturing increases in landing distance. For example, an increase in the minimum landing distance (for a dry runway).

- Directional Control Runway; concerned with changes in the ability to steer the airplane on the ground. For example, a reduction in the ability to perform low-speed steering.

- Fire Detection/Extinguishing; concerned with changes in the ability to detect and extinguish an on-board fire. For example, a change in the ability to extinguish fire in a specific region of the airplane.

- Surveillance; concerned with changes in the ability to detect external threats. For example, a change in the ability to alert on windshear.

- Fuel Supply; concerned with the ability to get fuel to the engines. For example, a change in the ability to get fuel to an engine.

We decided to supplement this description of capabilities with descriptions of the state of essential airplane resources

- Electric Power; concerned with the ability to provide electrical power.

- Hydraulic Power; concerned with the ability to provide hydraulic power.

- Pneumatic Power; concerned with the ability to provide pneumatic power.

- Equipment Cooling; concerned with the ability to cool equipment.

- Engine State; concerned with the state of each engine. 
These capability statements address both "unable to perform" and "not authorized to perform" (from a regulatory perspective). For example, if an airplane loses one of two GPSs, it is not authorized to fly an RNAV approach, but it would still be able to fly that approach, and a flight crew might be in such a desperate situation that they need to proceed. Therefore, in showing changes to capabilities, the display uses "Unable" or "Fail" to show the capability is lost because of equipment or system failures and uses "Not Authorized" to indicate that a regulatory requirement is not met but the capability may still be available. We will continue to evaluate how well this language conveys the appropriate understanding to pilots.

\section{Maneuvering}

Another major component of airplane capability is the airplane's ability to maneuver, and we have separated this display out of the larger set of capabilities. This display identifies losses or degradations to basic airplane maneuverability, such as a reduced turn rate, a limitation on airspeed, or a limit in the ability to slow the airplane. This display also identifies losses of automated support for aviating, such as envelope protections.

\section{Operational Limitations by Phase of Flight}

Airplane system failures can not only lead to changes in the airplane's ability to perform essential functions; these failures can also change the operational limitations on the airplane for continued safe flight and landing. The flight crew needs to understand the ways in which they should continue operating so they do not create additional problems for the airplane. Traditionally, non-normal checklists identify necessary changes that are tied to failures; e.g., use flaps 20 and Vref +20 on final approach.

We have developed a display that allows the flight crew to have awareness of and get access to all these operational limitations by phase of flight. A phase-of-flight organization is not typically found in current airplanes but fits best with operational requirements.

\section{E. Mission Risks}

Each flight (mission) has a set of risks associated with it, some anticipated and some that emerge during the course of the flight. Indeed, the shift in pilot training to a Threat and Error Management (TEM) framework [1] highlights the fact that there is a range of risks tied to each mission. This display integrates the set of mission risks to ensure that the flight crew can maintain awareness of the entire set for mission-planning. The display captures risks tied to the flight crew, the environment, and the airspace/infrastructure. Some of these are captured by the system and others may need to be entered by the flight crew.

\section{An Example Scenario}

The following illustration, taken from a recent NASA report [2], shows how these displays might appear. The case is an AC Bus failure that affects a number of airplane systems; electrical system failures can have far-flung effects. In this case, the AC bus failure leads to a number of downstream component failures. Although this illustration is not an actual case from a specific airplane, it accurately captures typical consequences from a bus failure.

For this scenario, the commercial jet transport is flying to Oklahoma City (KOKC); it is currently in cruise and cleared to fly the RNAV arrival and approach to runway $35 \mathrm{R}$. The initial mission overview display, captured in Figure 1, indicates compatibility with the mission prior to the bus failure. The top of the display shows the basic elements of the mission; the use of white for coding shows that the airplane is compatible with these mission requirements. 


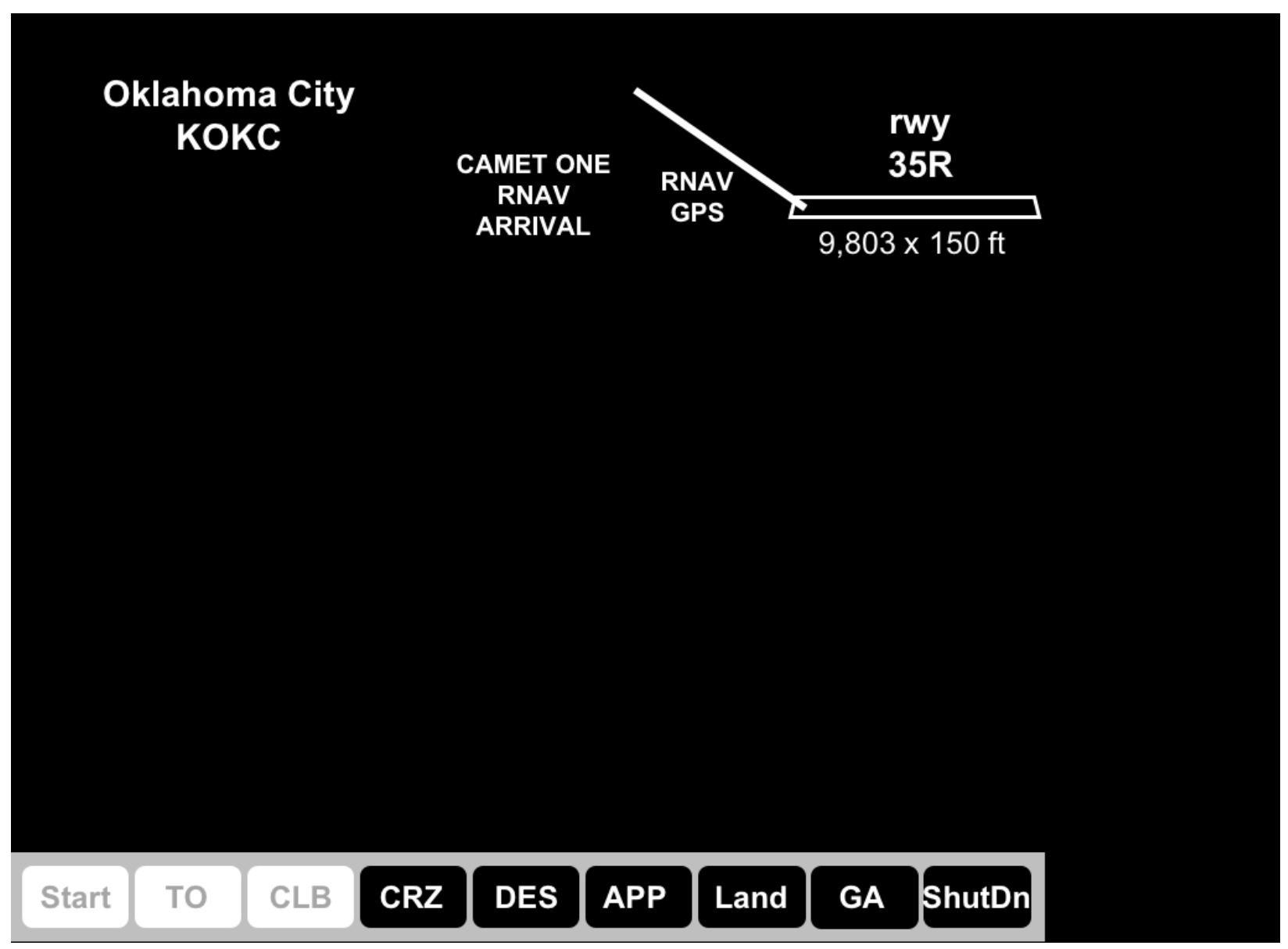

Fig. 1 Initial mission compatibility display

The strip of phase-of-flight labels at the bottom of the display keeps track of any changes to the airplane's operational limitations. At this point, there are no changes; all is normal.

When the AC bus failure occurs, it produces these 14 alert messages in the simulated airplane:

- AC BUS 1 OFF; AUTOTHROT DISC; ENG 1 EEC MODE ; FUEL PUMP 2; FUEL PUMP 3; WNDSHR ALERT SYS; GND PROX SYS; HEAT L TAT; OUTFLOW VLV L; HEAT L AOA; WINDOW HEAT L; HEAT P/S CAPT; AUTOBRAKE; ELEC UTIL BUS L

As stated above, these types of alert messages are typical of what would occur in a modern jet transport. The primary effects are on the functionality of autothrottle, fuel pumps, alerting systems, probe heaters, environmental control, and the autobrake. Also, LNAV and VNAV are lost. This list gets translated to the mission overview as shown in Figure 2. 


\section{Oklahoma City}

KOKC

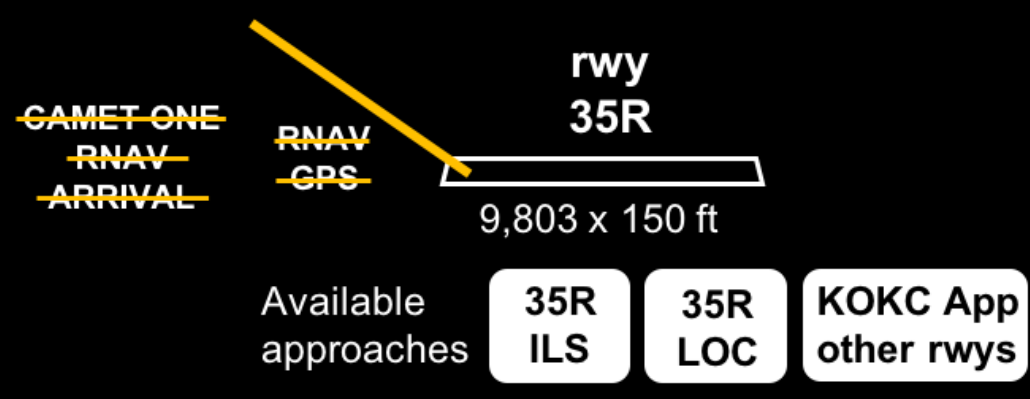

- LNAV + VNAV unable

AutoFlight

- Autothrottle unable

- GND PROX alert may not occur

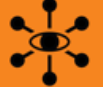

Surveil- • WINDSHEAR alert lance may not occur

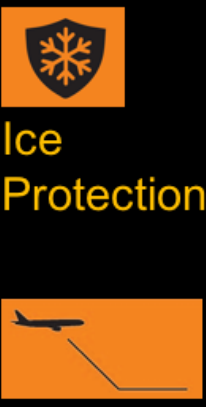

Approach Access
- Avoid icing conditions

- RNAV GPS approach not authorized

- RNAV GPS arrival not authorized

\section{Start TO CLB CRZ DES APP Land 1}

Fig. 2 After the AC Bus failure.

Because LNAV and VNAV are lost and these capabilities are required to compute a lateral and vertical path on board, the airplane is not able to fly the RNAV arrival and approach. The other available approaches to this runway are shown and can be selected. The airplane capabilities section of the display shows the four functional areas that have been affected: autoflight, surveillance, ice protection, and approach access. This is a subset of the larger set of airplane capabilities, and, ideally, aids the flight crew in making sense of what has been affected. Next, note that there have been changes to the operational limitations or deviations for four phases of flight. The display also indicates how many limitation/deviation items there are in each phase of flight. By clicking on that area of the display, you can navigate to another display (Figure 3 ) that provides details on these limitations. 


\section{Descent}

Do not exceed 250 kts below $10,000 \mathrm{ft}$ [WINDOW HEAT L]

\section{Approach}

Manual braking is required on landing

[AUTOBRAKE]

Require RVR $>4000$

[MALSR inop]

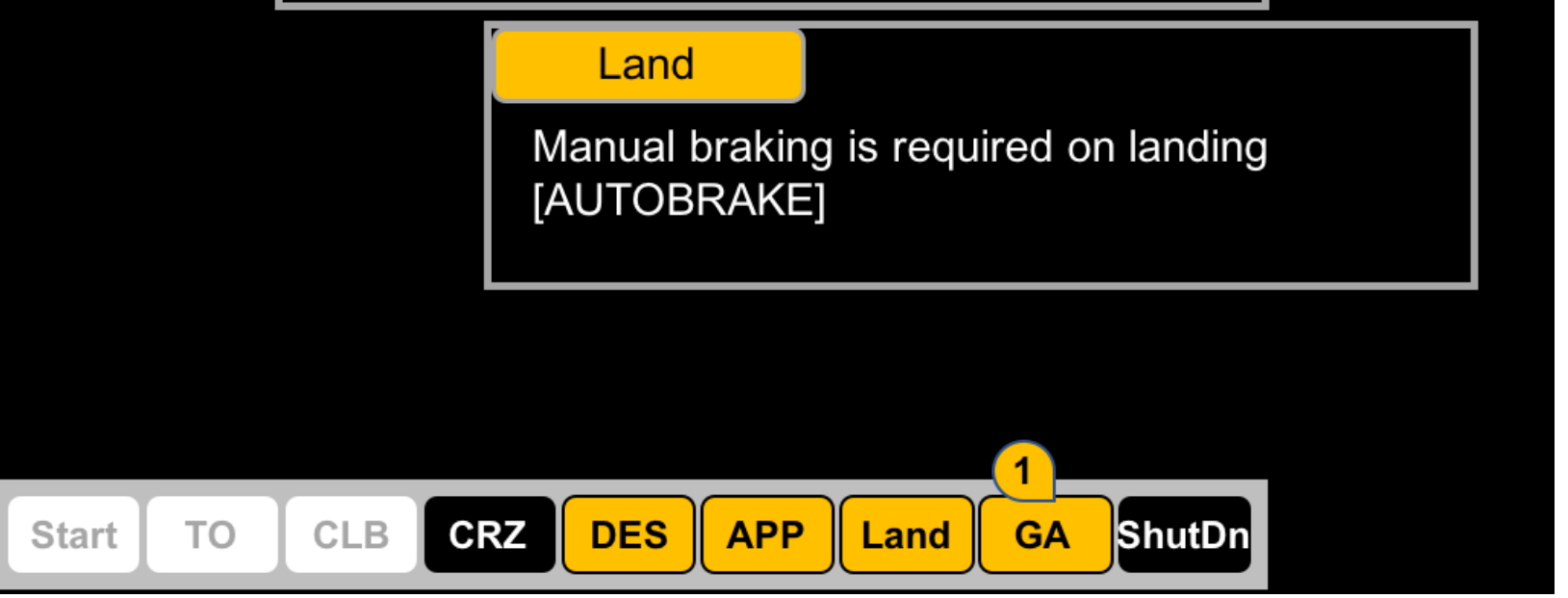

Fig. 3 Operational limitations display.

The display does not expand every phase of flight. In this case, it details three of them; other phases can be selected for viewing. Note that the MALSR inop issue (a loss of runway lighting) is from a NOTAM (notice to airmen).

Flight crew application of non-normal checklists (NNCs) can restore the autothrottle, but all the other losses remain after all NNCs have been performed. After the flight crew switches to the ILS approach, the mission overview display looks like Figure 4. 


\section{Oklahoma City}

KOKC

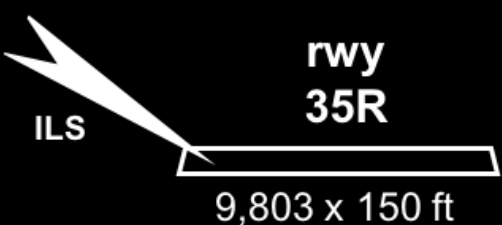

$9,803 \times 150 \mathrm{ft}$

- LNAV + VNAV unable

Auto-

Flight

Ice

- Avoid icing conditions

Protection

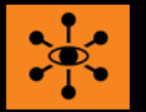

- GND PROX alert may not occur

Surveil- • WINDSHEAR alert lance may not occur

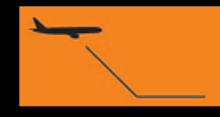

Approach

Access
- RNAV GPS approach not authorized

- RNAV GPS arrival not authorized

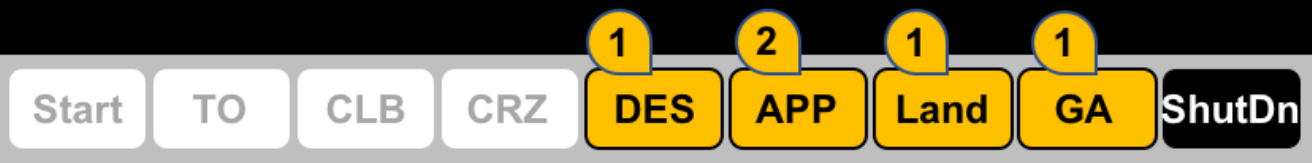

Fig. 4 After restoration of autothrottle.

The flight plan for the ILS approach is now compatible with the airplane's capabilities. The operational limitations remain to be managed.

The last event we created for this scenario was for the weather at KOKC to change; specifically, thunderstorm activity nearby the airport. This weather information is picked up and used to generate a message because of the airplane's particular vulnerabilities (icing and windshear alerting) (see Figure 5). 


\section{Oklahoma City}

KOKC

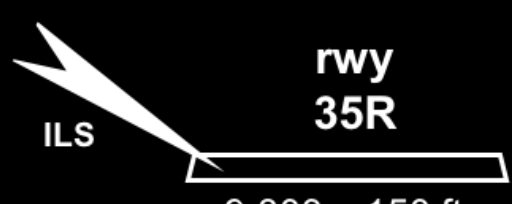

Icing conditions exist $\quad 9,803 \times 150 \mathrm{ft}$

Windshear potential exists

LNAV + VNAV
unable

Auto-

Flight

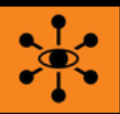

- GND PROX alert may not occur

Surveil- • WINDSHEAR alert

lance may not occur

- Avoid icing conditions

Ice

Protection

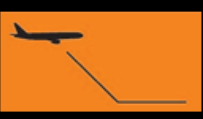

Approach Access
RNAV GPS approach not authorized

RNAV GPS arrival

not authorized

\section{1 \\ Start \\ TO \\ CLB \\ CRZ \\ DES \\ 2 \\ 1 \\ 1 \\ APP Land \\ GA \\ ShutDn}

Fig. 5 After weather information is added.

A remaining design issue is the combination of

- vulnerability to a hazard/threat (due to degradation or loss of a capability);

- the presence of the hazard/threat

The primary question is, "Is there value in explicitly indicating the combination of the two?" That is, is it enough to identify a lost capability (icing protection) and separately the presence of icing conditions? Or, should the mission compatibility display also explicitly identify the combination?

\section{Integration with the Existing Flight Deck}

We should note that not all of this information is new. Some of it exists currently - e.g., operational limitations, some maneuver limitations, loss of autoland - in many of the current flight deck interfaces or in the procedures provided with the airplane. We are both defining new information and organizing the larger sets of information to better support operational decision-making. 
The prototype development allows us to prompt pilot reviews and evaluate performance in simulated tasks to further refine these displays and compare this presentation to the current state of the art. Some of the issues we are continuing to explore are

- Currently, this display identifies only those capabilities that are degraded or lost. Remaining capabilities are not displayed, aligning with a "quiet, dark" interface philosophy. Is "quiet, dark" appropriate for the capabilities display or is there value in knowing the status of the full set of capabilities?

- Should all affected airplane capabilities be displayed or should we limit the display to just those that are relevant to the current mission?

- Should we organize as much as possible according to phase of flight or should limitations be displayed in a more enduring way?

- Should the types of information proposed here be provided on a separate flight deck display, integrated into existing displays, or would it replace existing formats?

We hope to begin to address these questions and others in our future work.

\section{Summary and Conclusions}

The objective of our work is developing and refining flight deck displays to support managing airplane system failures. The inevitable push toward more complex and interconnected airplane systems will lead to a significant gap between the information presented by the system (as airplane system component failures) and the flight crew's ability to use it for operational decision-making. Other industries, such as nuclear power, developed displays in the 1980s to better support operators in understanding how well operational objectives were being met [3]; aviation has lagged behind. This paper reviewed a set of display prototypes that we have developed to embody our ideas about presenting airplane capabilities. Further, there is an opportunity to better integrate information on the operational environment and airspace infrastructure into these displays to better support a range of operational decisions.

\section{References}

[1] Helmreich, R. L., Klinect, J. R., and Wilhelm, J. A. 'Models of threat, error, and CRM in flight operations,' Tenth International Symposium on Aviation Psychology, Columbus, OH, 1999, pp. 677-682.

[2] Mumaw, R.J., Feary, M., Fucke, L., Stewart, M., Ritprasert, R., Popovici, A., and Deshmukh, R. (2018). "Managing complex airplane system failures through a structured assessment of airplane capabilities," NASA/TM-2018-291775, 2018.

[3] Woods, D.D., Wise, J.A., \& Hanes, L.F. (1982). "Evaluation of safety parameter display concepts," EPRI NP2239 . 